\title{
Selective adaptation with reversible figures: Don't change that channel
}

\author{
THOMAS C. TOPPINO and GERALD M. LONG \\ Villanova University, Villanova, Pennsylvania
}

\begin{abstract}
An adaptation-test paradigm was used in two experiments examining processes underlying the perceived reversals of a rotating Necker cube. Adaptation and test cubes were either the same or different with respect to their visual fields of presentation (Experiment 1) or their sizes (Experiment 2). Results of both experiments indicated that, following subjects' adaptation to a different cube, reversal rate of the test cube did not differ from that obtained without prior adaptation experience. In contrast, reversal rate of the test cube was elevated following adaptation to the same cube. Additional findings of Experiment 1 were that a test cube presented to the same visual field as the adaptation cube yielded a higher reversal rate than did a simultaneously presented cube in the opposite visual field. Also, the reversal rate of one cube was not influenced by the simultaneous presentation of a second cube. Results of both experiments were interpreted in terms of the fatigue and recovery of multiple, largely independent, localized neural channels. Thus, the results tie reversible-figure illusions to other visual phenomena thought to involve similar fatigue processes within localized visual channels (e.g., tilt, motion, and size aftereffects).
\end{abstract}

Reversible figures make up a well-known class of visual illusion in which a stimulus pattern appears to alternate spontaneously between different perceptual organizations or interpretations during continuous viewing. Familiar examples of this kind of illusion include the Necker cube, Rubin's vase/faces, the Schröder staircase, the Mach folded card, Fisher's man/girl, Boring's young girl/old woman, and the Maltese cross (see Attneave, 1971, for examples of these and other reversible figures). The curious multistable character of reversible figures has intrigued students of visual perception for over a century and a half, since an early report of such a phenomenon was published by Necker in 1832 (cited in Boring, 1942). The continued interest in these illusions reflects the assumption that understanding the multistability of reversible figures will provide insight into basic perceptual mechanisms.

Aside from the fact that the perceptual interpretation of reversible figures appears to fluctuate, the most wellestablished phenomenon associated with reversible figures is that the perceptual reversals tend to occur more and more rapidly over an extended viewing period. In numerous studies, the number of reversals per unit of time has been found to be an increasing negatively accelerated function of viewing time (e.g., Babich \& Standing, 1981; Brown, 1955; Cohen, 1959; Howard, 1961; Long, Top-

\footnotetext{
This research was supported in part by Grant 1 R03 MH38197-01A1 from the National Institute of Mental Health to the first author and by Grant 1 R03 EY05846-01 from the National Eye Institute to the second author. Various aspects of the data contained in this article were presented at the annual meeting of the Psychonomic Society in San Antonio, November 1984, and at the annual meeting of the Association for Research in Vision and Ophthalmology in Sarasota, May 1986.

Requests for reprints should be sent to Thomas C. Toppino, Department of Psychology, Villanova University, Villanova, PA 19085.
}

pino, \& Kostenbauder, 1983; Price, 1969a, 1969b; Spitz \& Lipman, 1962). Although several theories have been offered to account for the basic characteristics of reversible figures, most incorporate one of two major sets of theoretical mechanisms. In one set, reversible-figure illusions are explained in terms of the alternating satiation (fatigue) and recovery of neural structures, whereas, in the second set, the illusions are explained in terms of perceptual decision making (or hypothesis testing) and perceptual learning.

Theorists proposing fatigue/recovery mechanisms assume that different cortical organizations underlie each perceived variation of a reversible figure. These underlying organizations are assumed to involve mutually inhibitory processes such that only one perspective of a reversible figure can be seen at a time. Perceptual fluctuation is thought to be the phenomenal result of a cyclic process of fatigue and recovery. With extended viewing, the cortical organization underlying one alternative percept satiates or fatigues and is supplanted by a new, fresher cortical organization underlying the other perspective. The second organization also fatigues with further viewing and is replaced eventually by the first organization, which has had time to recover. This recovery is assumed to be incomplete, however, so that satiation and perceptual reversal then occur more quickly. Over a long viewing period, successive reversals should occur more and more rapidly until some relatively constant rate of satiation and recovery is attained, thus accounting for the negatively accelerated function relating number of reversals to viewing time. Although satiation theories were formulated originally in terms of Gestalt assumptions about the physiological processes underlying perception (e.g., Kohler \& Wallach, 1944), more recent investigators (e.g., Harris, 1980; Long \& Toppino, 1981; Long et al., 1983; Palmer \& Bucher, 
1981; von Grünau, Wiggin, \& Reed, 1984) have related satiation effects to the fatiguing of neural "channels" that often are hypothesized to constitute the visual system (e.g., Braddick, Campbell, \& Atkinson, 1978; Regan, 1982).

Theorists proposing learning/decisional mechanisms to account for reversible-figure illusions emphasize the operation of active processes that usually are associated with the use of limited attentional resources. According to this view, our perceptions are the result of hypothesis-testing or problem-solving processes (e.g., Gregory, 1970; Rock, 1975). The perceptual alternations of a reversible figure are attributed to a cyclical decision process in which the perceptual system vacillates between two equally acceptable "solutions" to the perceptual puzzle, attending first to one and then to the other. By incorporating aspects of earlier learning-based theories (e.g., Ammons, 1954; Ammons, Ulrich, \& Ammons, 1959), decisional theories explain the increase in reversals with extended viewing as the result of learning from perceptual experience. The fact that the number of reversals is an increasing negatively accelerated function of viewing time is thought to reflect a standard learning curve for the perceptual response involved.

Both fatigue/recovery and decisional/learning theories have received some empirical support. For example, the former theories have been supported by studies showing that viewing an unambiguous version of a normally ambiguous figure results in subjects' perceiving the alternative interpretation when the standard reversible figure is subsequently presented (e.g., Carlson, 1953; Harris, 1980; Hochberg, 1950; Virsu, 1975). On the other hand, several findings seem more consistent with decisional/learning theories. For example, subjects can control reversal rates voluntarily to some extent (e.g., Pelton \& Solley, 1968), and, unless subjects know that the stimuli are reversible, they may fail to perceive reversals at all (Girgus, Rock, \& Egatz, 1977). In addition, practice effects have been obtained in which reversal rate improves from viewing session to viewing session even when relatively long delays (e.g., a week) separate sessions (e.g., Long et al., 1983).

In the present research, we further examined the mechanisms underlying reversible-figure illusions by contrasting fatigue and decisional theories with respect to two closely related issues. The first issue concerns the local or global nature of the underlying mechanism. By hypothesizing that fatiguing neural channels play a central role in the perception of reversible figures, recent satiation theories imply the existence of multiple, largely independent processes (channels), each responsive to a specific type of stimulation in a localized region of the visual field. In contrast, decisional explanations seem to involve a mechanism having a more general, relatively global domain of operation. The second issue concerns the degree to which the underlying mechanisms are subject to attentional limitations. Fatigue theories imply that the multiple neural channels are passive, data-driven processes that operate automatically. That is, they are triggered by appropriate retinal stimulation and require little or no attentional capacity. Thus, multiple channels are capable of operating in parallel with little or no interference. In contrast, decisional theories imply the involvement of a global decisional mechanism that is active, is at least partially conceptually driven, and uses limited attentional resources.

Our characterization of fatigue theories as involving local processes with minimal attentional limitations is consistent with a variety of studies dealing with the existence and operation of fatigable visual channels (e.g., Frisby, 1980). However, our characterization of decisional theories as involving relatively global processes that use limited attentional resources may require further discussion. This characterization is based on the following considerations that have been noted also in our previous work (Long \& Toppino, 1981; Long et al., 1983). First, according to decisional theories, different interpretations of a single reversible figure are seen successively because the related perceptual decisions are made serially. If these decisions could be made in parallel, simultaneous perception of both interpretations of a single reversible figure would be possible. Second, any serial decision mechanism would appear to be limited in the number of decisions per unit of time, although the practical extent of the limitation depends on the speed with which successive decisions can be made. Third, it is recognized that, rather than assuming a global decisional mechanism, one could postulate the existence of multiple serial decision makers (one for each of an indefinitely large number of figures throughout the visual field) that function in parallel without interference. However, the notion of innumerable trialand-error decisional processes operating (and undergoing perceptual learning) largely independently of one another is neither very appealing nor very consistent with the general conceptualization of such processes. Furthermore, if one chooses a decisional model with such unwieldy characteristics, the functional distinctions between decisional and fatigue models begin to blur.

The first issue concerning the involvement of local or global processes was addressed in both experiments presented here through the use of the popular selective adaptation paradigm (see Frisby, 1980). Observers viewed a rotating Necker cube for a 2-min adaptation period, followed immediately by a 2 -min test period. The cube was either identical in the adaptation and test phases, or it was changed so that different cortical channels would mediate its perception. In Experiment 1, this change involved shifting the rotating cube from one visual field to the other. In Experiment 2, the size of the rotating cube was altered. If local processes of the sort implied by satiation theory are operative, the effect of exposure during the adaptation phase should transfer to the test phase only when stimuli (and underlying cortical channels) are identical in both phases of the experiment. The effect of the more global processes associated with decisional theories would not be expected to be so limited.

The second issue concerning the attentional characteristics of the processes underlying the perception of reversible figures was addressed in Experiment 1 by having 
some observers simultaneously view two cubes, one to the left and the other to the right of fixation. If reversals in the two cubes are mediated by independent neural channels that operate automatically and in parallel, reversal rate for a given cube should be unaffected by the number of cubes being viewed. However, from the viewpoint of decisional theory, a serial decisional mechanism would have to divide its decision-making time between the two cubes. Thus, the reversal rate for each of two cubes viewed simultaneously should be lower than for either cube viewed alone.

\section{EXPERIMENT 1}

Evidence for multiple, local processes mediating the fluctuations of reversible figures was provided by a recent series of studies by von Grünau et al. (1984). Using stationary Necker-cube-like figures, these investigators adapted observers to an unambiguous version of their figure and then transferred them to an ambiguous version. An adaptation effect in which observers perceived the perspective of the ambiguous figure to be the opposite of the unambiguous adapting figure was obtained only when both figures were presented to the same portion of the visual field. Potentially converging evidence can be found in other studies that have adapted subjects to an ambiguous figure presented to one visual field (e.g., left) and then transferred them to an identical ambiguous figure presented to the opposite visual field (e.g., Babich \& Standing, 1981; Cohen, 1959; Howard, 1961; Spitz \& Lipman, 1962). Unfortunately, there is at least some reason to question the results of all of these latter experiments.

Howard (1961) found that observing a rotating cube in one visual field did not influence the perceived reversal rate of a cube that subsequently was presented in the opposite visual field. However, Howard did not include a "same-adaptation"' condition in which the first and second cubes (adaptation and test cubes, respectively) were presented to the same region of the visual field. Such a comparison condition is necessary to conclude that the spatial relationship between adaptation and test cubes is the critical factor producing the results and not some extraneous feature of the procedure. Other investigators using stationary Necker cubes did include a same-adaptation condition in their experiments. However, their results were inconclusive because either they used somewhat different viewing conditions in the same- and differentadaptation conditions (e.g., Babich \& Standing, 1981; Spitz \& Lipman, 1962) or their observers tended not to see reversals during the adaptation phase of the experiment, making it unclear whether the observers actually underwent adaptation (Cohen, 1959).

One purpose of the present experiment was to investigate the effects of presenting adaptation and test figures (rotating Necker cubes) to the same or different visual fields while avoiding the problems that afflicted previous studies. If reversals are mediated by the fatigue and recov- ery of multiple, localized channels in the visual system, the reversal rate of the test cube should be elevated after adapting to a cube in the same visual field but not after adapting to a cube in the opposite visual field. If reversals are the result of a global decision-making mechanism, however, the reversal rate of the test cube should be elevated regardless of whether adaptation involved a cube in the same or the opposite visual field. The increase in reversal rate with experience is assumed to be due to perceptual learning. There is little reason to believe that a decision maker would undergo perceptual learning while attending to a figure presented in one visual field during adaptation but would be unable to apply that learning when attending to an identical figure appearing in the opposite visual field.

The second purpose of Experiment 1 was to examine how the reversal rate of a rotating Necker cube would be affected by a second rotating Necker cube presented simultaneously in the alternate visual field. Such a manipulation would address the question of whether perceived reversals reflect the operation of largely passive, independent mechanisms functioning in parallel or an active, limited-capacity mechanism operating serially. This line of investigation stems from observations reported by Long and Toppino (1981) and Long et al. (1983). Although numerous researchers had reported that simultaneously presented ambiguous figures tend to reverse separately (e.g., Gillam, 1972; Howard, 1961; Mefferd et al., 1966; von Grünau et al., 1984; Washburn, Mallay, \& Naylor, 1931), Long and Toppino made the point that these independent reversals seem to be quite consistent with the operation of multiple, localized neural channels that operate passively and in parallel. However, the phenomenon seems to be less easily reconciled with a decisional interpretation. To account for the fact that each of several reversible figures can fluctuate largely independently of one another, one might postulate that separate decisional mechanisms underlie the alternating perceptions of each figure. However, this assumption is incompatible with the global character usually attributed to decisional mechanisms and blurs the distinctions between these mechanisms and fatigue processes. Alternatively, one could account for the separate reversals of multiple figures by assuming that a global, serial, decisional mechanism attends first to one figure and then to another. However, as Long et al. noted, this would require a serial mechanism to divide its time among the simultaneously presented figures. As a result, the reversal rate for any one figure should be slower in the multiple-figure task than in the usual singlefigure task.

The latter prediction was tested but was not confirmed by Long et al. (1983). Observers reported perceived reversals for a single rotating Necker cube or for both of two simultaneously viewed rotating Necker cubes. In accordance with the prediction of satiation theory and contrary to the prediction of decisional theory, the reversal rate for a given cube was uninfluenced by the presence or absence of a second cube. However, there are two rea- 
sons why this finding may be viewed as less than conclusive. First, the finding was a null effect and certainly requires replication to bolster our confidence in the outcome. Second, there was no independent evidence (other than subject reports) that observers who viewed two cubes in the Long et al. experiment perceived the cubes to reverse independently. If, in fact, the cubes always appeared to rotate in the same direction and to reverse in unison, one might hypothesize from the perspective of decisional theory that each double reversal was due to a single global decision. In this case, decisional theory might not expect the reversal rate for each of two simultaneously presented cubes to differ from that of a cube presented in isolation.

In Experiment 1, some subjects adapted to a single cube presented to the left or to the right visual field and then viewed two cubes, one in each visual field, during the following test phase. This allowed a replication of the finding reported by Long et al. (1983) under potentially clearer conditions. If multiple independent channels mediate reversals, reversal rate should differ between the two cubes viewed during the test phase. The cube that is the same as the adaptation-phase cube should reverse more rapidly. Moreover, if the localized channels underlying the perception of each cube can operate in parallel without interference, reversal rate of either cube should not differ from the reversal rate of a single cube with a comparable adaptation history. Turning to decisional theory, it is not clear how one could predict that the reversal rate of the two test-phase cubes would differ. However, such a difference in reversal rate would clearly indicate that the two cubes were not always reversing in unison. Under these circumstances, a global mechanism that makes perceptual decisions serially would have to share its decisionmaking time between cubes. This leads to the prediction that the reversal rate for each of the two cubes would be slower than for a single cube viewed under comparable conditions.

\section{Method}

Subjects. Fourteen males and 14 females were randomly assigned to serve as subjects in each of six viewing conditions. Only righthanded individuals were recruited and allowed to participate in the experiment. All 168 subjects ( 84 males and 84 females) were students at Villanova University and participated for partial fulfillment of the requirements of a general psychology course.

Apparatus. The apparatus was the same as that used previously by Long et al. (1983). The subjects were seated $1 \mathrm{~m}$ from a 125 $\times 265$ mm screen, consisting of a translucent pane of glass covered by a piece of medium-weight white paper. Either one or two shadows of a Necker cube ( $45 \mathrm{~mm}$ on a side) were rear projected on the screen by using either one or two 150-W light bulbs positioned $95 \mathrm{~cm}$ apart and $88 \mathrm{~cm}$ from the Necker cube. The luminance on the white screen was $1.0 \mathrm{fL}$. The shadows of the cubes were projected so that their centers were $42.5 \mathrm{~mm}$ to the right and/or left of a 10-mm black fixation point on the screen. When a fullfront face was displayed, the size of the projected cubes was $47 \mathrm{~mm}$ per side, which corresponds to a visual angle of approximately $2.7^{\circ}$ at a distance of $1 \mathrm{~m}$. The cube rotated at a constant rate of 10 rotations/minute. The subjects placed their index fingers on standard telegraph keys. They reported each perceived reversal of the left and/or right cube by pressing the left and/or right telegraph keys, respectively. The telegraph keys were connected to Foringer equipment and electromechanical counters so that each response corresponding to a perceived reversal was recorded automatically.

Procedure. Each subject participated individually in a 30-min session. At the outset, the subject was given a 1-min observation period to adapt to the apparatus and to become familiar with reversals of both a stationary and a rotating Necker cube. After experiencing reversals, the subject was given a 2 -min rest period to allow potential satiation effects from this initial viewing period to dissipate. Then the subject received 4-min viewing blocks, each separated by a 2 -min rest period. The first 2 min of each 4-min block constituted the adaptation phase, during which the subject viewed the shadow of one rotating Necker cube. The remaining 2 min constituted the test phase, during which the subject observed the shadows of one or, in some conditions, two rotating Necker cubes. The display was completely darkened for about $1 \mathrm{sec}$ between the adaptation and test phases. The subject was instructed to observe the display passively during each 4-min block and to report each reversal that occurred by depressing the appropriate telegraph key. The subject was instructed to keep his/her eyes on the fixation point during all viewing periods, but no independent check on fixation was made. The experimenter recorded the number of reversals reported within each of the four 30-sec intervals during the 2-min adaptation phase and the 2-min test phase of each 4-min block.

Viewing conditions. Each subject served in one of the following six viewing conditions, which were differentiated by what subjects observed and counted in the adaptation and test phases of each 4-min block:

Left-Left $(L-L)$. Subjects were presented a single rotating cube in the left visual field during both the adaptation and the test phases.

Left-Right $(L-R)$. Subjects were presented a single rotating cube in the left visual field during the adaptation phase and a single rotating cube in the right visual field during the test phase.

Right-Left $(R-L)$. Subjects were presented a single rotating cube in the right visual field during the adaptation phase and a single rotating cube in the left visual field during the test phase.

Right-Right $(R-R)$. Subjects were presented a single rotating cube in the right visual field during both the adaptation and test phases.

Left-Both $(L-B)$. Subjects were presented a single rotating cube in the left visual field during the adaptation phase and two cubesone in each visual field-during the test phase.

Right-Both $(R-B)$. Subjects were presented a single rotating cube in the right visual field during the adaptation phase and two cubesone in each visual field-during the test phase.

It should be noted that in the latter two conditions (L-B and RB), subjects counted perceived reversals of both cubes during the test phase by pressing the left and/or right key as appropriate.

\section{Results and Discussion}

The results were analyzed by a series of planned comparisons. In all analyses, each subject's data were collapsed across the four 4-min viewing periods, because primary interest centered on the effect of successive 30sec intervals within each viewing block and on the relationship between the adaptation and test phases within each viewing block. Data also were collapsed across sex of the subjects, because preliminary analyses indicated that this factor did not reliably influence performance in this experiment.

Effect of viewing time. The literature indicates that the number of perceived reversals of an ambiguous figure increases as a negatively accelerated function of viewing time. To determine whether our procedures produced this classic effect, the data of subjects in conditions $\mathrm{L}-\mathrm{L}$ and 
R-R were submitted to a 2 (cube: left vs. right) $\times 8$ (interval: successive 30 -sec intervals within a 4-min viewing period) ANOVA with repeated measures on the last factor. The only significant source of variance was the main effect of interval $[F(7,364)=21.08, p<.001]$. As can be seen from Figure 1, the number of reversals was an increasing negatively accelerated function of successive 30-sec intervals within a 4-min viewing block.

Adaptation effects. One issue addressed by the present research concerned how perceived reversals of a rotating Necker cube were influenced by adaptation to a cube appearing either in the same retinal location or in the opposite visual field. Because each hemifield projects to a different hemisphere, we decided to analyze the data separately for cubes presented to each hemifield.

In the first set of analyses, the number of perceived reversals obtained following adaptation to a cube in the opposite visual field (different adaptation) was compared with the number obtained without prior exposure to a cube during the viewing period (no adaptation). The data are presented in Figure 2. In the analysis of left-cube reversals, data for the different-adaptation condition were taken from the test phase of Group R-L and from the left test cube for Group R-B [denoted R-B(L)]. (The inclusion of data from one test cube in the R-B or the L-B groups is justified by later analyses assessing the reversal rate of a given cube as a function of the presence or absence of a second cube.) The data for the no-adaptation condition were taken from the adaptation phase of Groups L-L, L$R$, and L-B. These data were submitted to a 2 (adaptation: different vs. no adaptation) $\times 4$ (interval: successive 30 sec viewing intervals) ANOVA with repeated measures on the second factor. Results indicated that perceived reversals increased with successive viewing intervals $[F(3,414)=95.89, p<.001]$. However, the difference between the different-adaptation and no-adaptation conditions did not approach significance $[F(1,138)=1.16$, $p>.05]$, and the adaptation $\times$ interval interaction was

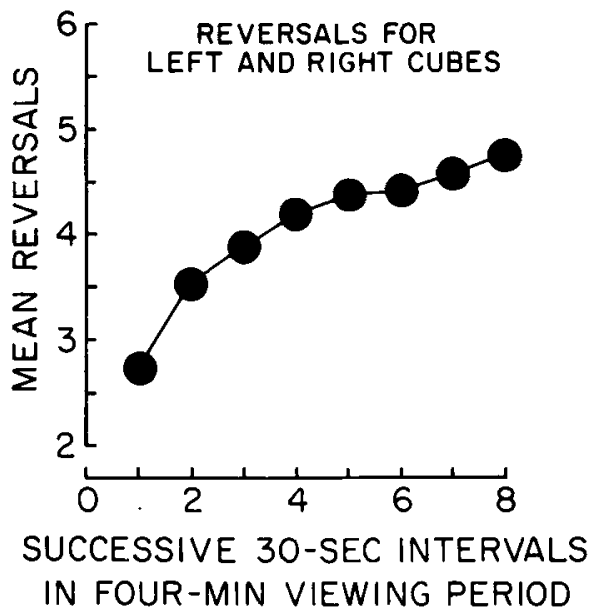

Figure 1. Mean reversals reported for a rotating Necker cube in Experiment 1 as a function of 30-sec intervals constituting a 4-min viewing period.
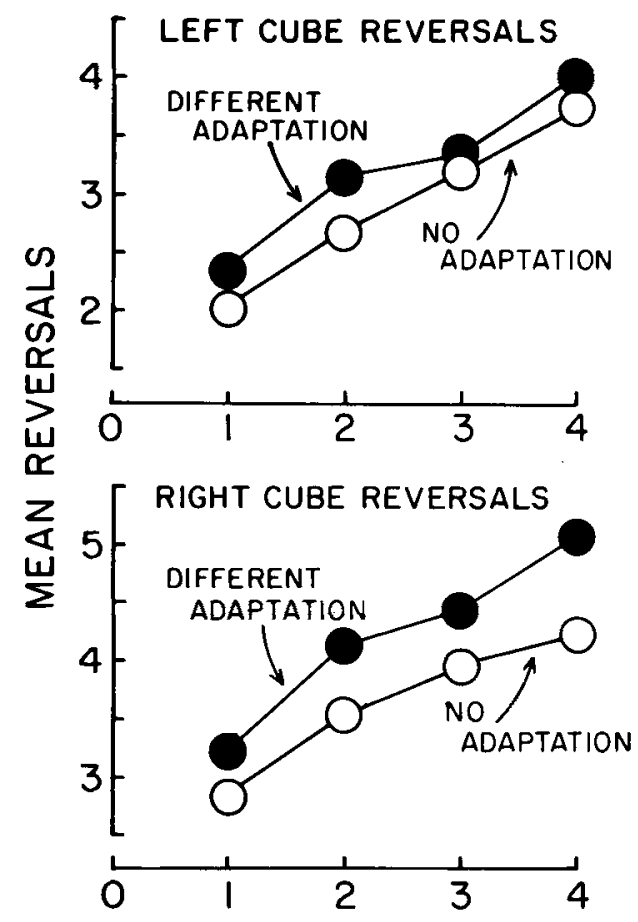

SUCCESSIVE 30-SEC INTERVALS

IN TWO-MIN TEST PERIOD

Figure 2. Mean reversals reported for a rotating Necker cube in Experiment 1 as a function of adaptation condition (different vs. no adaptation) and $30-\mathrm{sec}$ intervals constituting a 2 -min viewing period.

also unreliable $(F<1.0)$. In the analysis of right-cube reversals (bottom portion of Figure 2), data for the different-adaptation condition were taken from the test phase of Group L-R and from the right test cube of Group L-B [denoted L-B $(R)$ ]. The data for the no-adaptation condition were taken from the adaptation phase of Groups R-R, R-L, and R-B. These data were submitted to a second 2 (adaptation condition) $\times 4$ (interval) ANOVA with repeated measures on the second factor. The results were essentially the same as those for the left cube. Perceived reversals increased with successive viewing intervals $[F(3,414)=68.56, p<.001]$. However, there was no significant effect due to adaptation condition $[F(1,138)$ $=2.84, p>.05]$ or to the adaptation $X$ interval interaction $(F<1.0)$.

In the second set of analyses, the effect of adaptation to a cube in the opposite visual field (different adaptation) was compared to the effect of adaptation to a cube presented to same retinal location (same adaptation). The results are presented in Figure 3. The data for the different-adaptation conditions were the same as they were for the last two analyses. For the same-adaptation conditions, data for the left cube were taken from the test phase of Groups L-L and L-B(L), and data from the right cube were taken from the test phase of Groups $R-R$ and $R-B(R)$. Left- and right-cube data were each submitted to a 


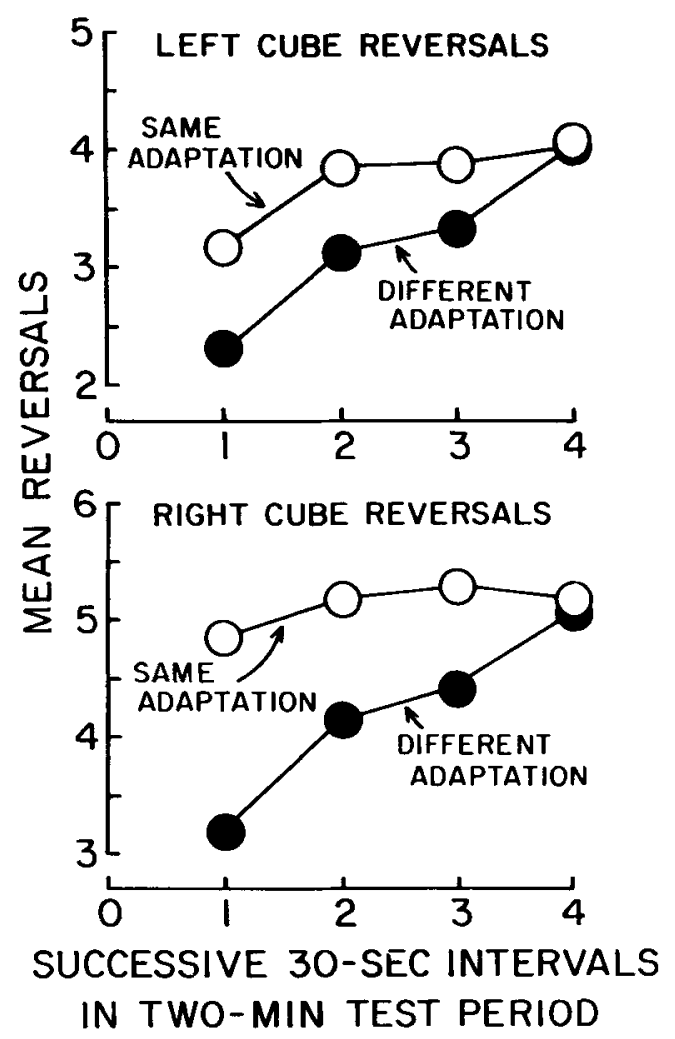

Figure 3. Mean reversals reported for a rotating Necker cube in Experiment 1 as a function of adaptation condition (same vs. different) and 30-sec intervals constituting a 2-min viewing period.

2 (adaptation: same vs. different) $\times 4$ (interval: successive 30-sec intervals during the 2-min test phase) ANOVA with repeated measures on the second factor. For the left cube (Figure 3, top), the main effect of interval indicated that the number of perceived reversals increased with extended viewing time $[F(3,330)=41.28, p<.001]$. More importantly, although the main effect of adaptation was not reliable $[F(1,110)=2.19, p>.05]$, there was a significant interval $\times$ adaptation interaction $[F(3,330)$ $=3.95, p<.01]$. Simple main effect analyses of the interaction indicated that in the first 30-sec interval of the test period, subjects in the same-adaptation condition reported more reversals than did subjects in the differentadaptation condition $[F(1,49)=4.54, p<.05] .{ }^{1}$ However, the reversal rate increased more rapidly with viewing time for the different-adaptation condition than for the same-adaptation condition. As a result, the disparity in perceived reversals between the same- and differentadaptation conditions failed to attain significance in the second 30 -sec interval $[F(1,149)=3.22, p<.10]$, or even to approach significance in the third and fourth intervals $[F \mathrm{~s}(1,149) \leq 1.98, p>.10]$. For the right cube (Figure 3, bottom), results were similar. The main effect of interval was reliable $[F(3,330)=20.78, p<.001]$. In addition, the effect of adaptation condition bordered on significance $[F(1,110)=3.78, p=.054]$, and the in- terval $\times$ adaptation interaction clearly was reliable $[F(3,330)=10.17, p<.001]$. The interaction took essentially the same form as it had for the left cube. Subjects in the same-adaptation condition reported significantly more reversals than did subjects in the different-adaptation condition in the first, second, and third 30-sec intervals $[F \mathrm{~s}(1,143)=18.19,7.13$ and 5.32 , respectively $]$, but not in the last 30-sec interval $(F<1.0)$ (see Note 1).

To summarize, during the adaptation period, reversal rate increased as a function of viewing time. If the subsequent test cube was presented to the same location in the visual field, the reversal rate for the test cube remained elevated at a near asymptotic level. If the test cube was presented to the opposite visual field, its reversal rate was no different from that obtained without prior exposure to an adaptation cube. That is, reversal rate was low at the outset of the test period and rose with viewing time toward asymptote. Consequently, the disparity in reversal rate between the same- and different-adaptation conditions was greatest at the beginning of the test period and diminished with additional viewing time as fluctuations in the different-adaptation condition approached their common asymptote.

These results are entirely consistent with the hypothesis that the fluctuations of reversible figures are due to the fatigue and recovery of multiple, independent neural channels, each sensitive to stimulation in a localized region of the visual field. As such, the results clarified the findings of several previous studies and confirmed the conclusions that had been suggested by these studies (e.g., Babich \& Standing, 1981; Cohen, 1959; Howard, 1961; Spitz \& Lipman, 1962). In contrast, these findings are not easily reconciled with the operation of a global decisional mechanism. If the very same process were to mediate perceived reversals for cubes presented in either the left or the right visual field, one would not expect adaptation effects to be limited by the location of stimulation in the visual fields.

Effect of simultaneously viewing two cubes. Subjects in Conditions L-B and R-B viewed one cube in the left or right visual field during adaptation and then viewed two cubes-one in each visual field- during the test phase. Thus, one cube was presented to the same retinal location as was the adaptation cube, whereas the other cube was presented to a different retinal location. An analysis was undertaken to determine whether the two test cubes differed from one another in terms of reversal rate. The test-phase data of Groups L-B and R-B were used in a 2 (adaptation: same vs. different) $\times 4$ (interval: successive 30-sec intervals during the 2-min test phase) ANOVA with repeated measures on both factors. The results, which are presented in Figure 4, mirrored the between-subjects analyses of adaptation effects. There was a significant main effect of interval $[F(3,165)=25.61$, $p<.001]$ and a significant interval $\times$ adaptation interaction $[F(3,165)=6.25, p<.001]$. Analyses of simple main effects indicated that the reversal rate was higher for the same-adaptation condition than for the different- 


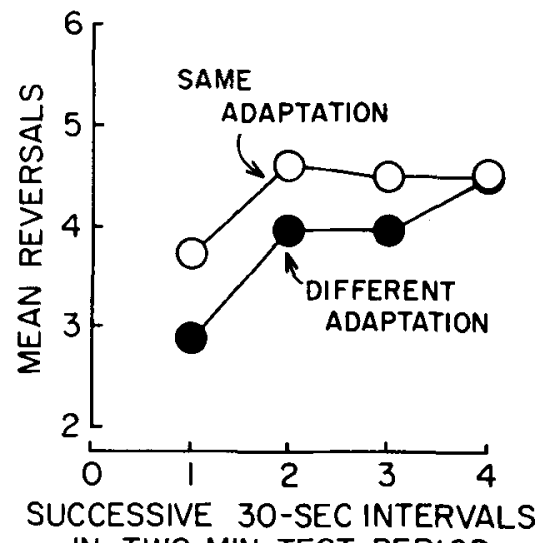

IN TWO-MIN TEST PERIOD

Figure 4. Mean reversals reported for two simultaneously viewed rotating Necker cubes in Experiment 1 as a function of same versus different adaptation and of 30 -sec intervals constituting a 2-min viewing period.

adaptation condition in the first and second 30 -sec intervals of the test period $[F s(1,220)=6.56$ and 4.52 , respectively], but not for the last two $30-\sec$ intervals $\left[F_{s}(1,220)\right.$ $\leq 3.26, p>.05$ ].

These results indicate the selective nature of the adaptation within a single observer at a given point in time. When the same observer views two cubes at once, the cube presented to the same retinal location as a prior adaptation cube will appear to reverse more rapidly than will a cube that is simultaneously presented to the opposite visual field. This kind of selective adaptation is exactly what one would predict from the hypothesis that reversals are mediated by multiple, independent neural channels. It is not so obvious how this finding would be explained in terms of decisional mechanisms. Putting this issue aside, however, the results clearly indicate that the simultaneously viewed cubes in this experiment were reversing separately from one another. Thus, the conditions were satisfied to test the prediction derived from decisional theory that the reversal rate of a given cube would be reduced by the simultaneous presence of a second cube.

To test the latter prediction, we examined how the rate of perceived reversals for a cube presented to one visual field was affected by the presence or absence of a second cube in the opposite visual field. Two analyses were conducted, one for the left cube and one for the right cube. Each analysis was a 2 (cube number: one vs. two) $x$ 4 (interval: 4 successive $30-\mathrm{sec}$ intervals) ANOVA with repeated measures on the second factor. All data were taken from the 2-min test phase of the 4-min viewing period.

The first analysis examined the effect of the number of cubes monitored by the subject on the perceived reversals of the left cube. For the two-cube condition, data were the perceived reversals of the left test cube by subjects in Groups $L-B$ and $R-B$ [denoted $L-B(L)$ and $R-B(L)$, respectively]. For the one-cube condition, data were the perceived reversals of the left test cube by subjects in Groups
L-L and R-L. These data are presented in the top panel of Figure 5. As can be seen, the number of perceived reversals for the left cube increased with successive 30 sec viewing intervals $[F(3,330)=38.32, p<.001]$, but was largely unaffected by whether or not subjects were also reporting perceived reversals of a second cube in the right visual field. The main effect of number of cubes was not significant $(F<1.00)$, and, although the cube number $\times$ interval interaction attained significance $[F(3,330)$ $=3.21, p<.05]$, simple main effect analyses of number of cubes for each interval revealed that the number of cubes had no reliable effect at any of the four intervals (all $F$ s $<1.00$ ).

The second analysis examined perceived reversals of the right cube as a function of the number of cubes. The relevant data are presented in the bottom panel of Figure 5. For the two-cube condition, the data were the perceived reversals of the right test cube by subjects in Groups L-B and R-B [denoted L-B(R) and R-B(R), respectively]. Corresponding data for the one-cube condition were the perceived reversals of the right test cube by subjects in Groups L-R and R-R. The ANOVA indicated that the number of perceived reversals increased as a function of successive 30 -sec viewing intervals $[F(3,330)=$ $21.06, p<.001$ ] but that there was no reliable effect of either the number of cubes $(F<1.00)$ or the number of cubes $X$ interval interaction $[F(3,330)=1.27, p>.05]$.

To summarize, as clearly indicated by the pattern of results reported in Figure 5 and supported by the statistical analyses, these data failed to support the prediction

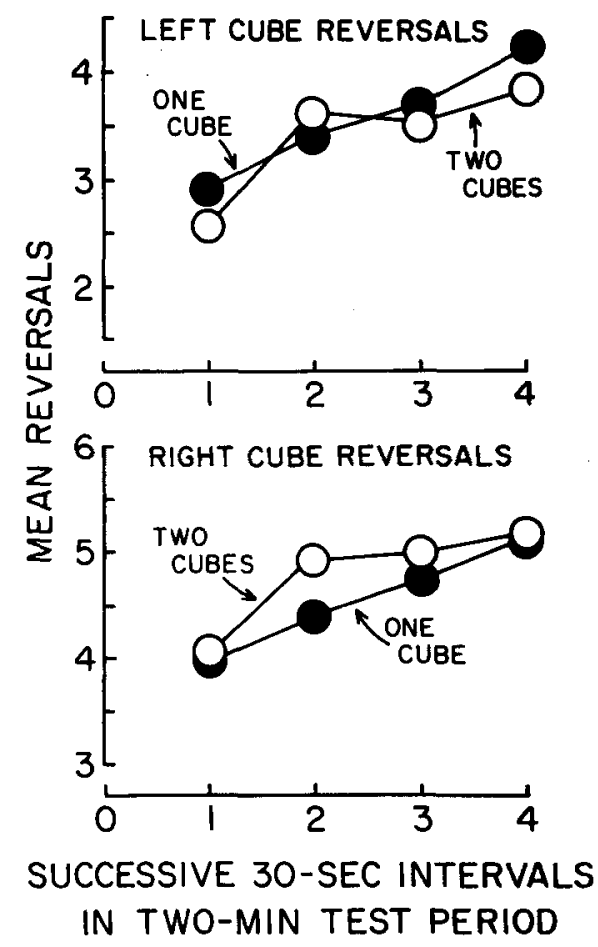

Figure 5. Mean reversals reported for a rotating Necker culbe in Experiment 1 as a function of the number of cubes being viewed (one vs. two) and 30-sec intervals constituting a 2-min vieving period. 
derived from decisional theory that reversal rate would be influenced by the presence of a second cube. These findings replicate the results of Long et al. (1983), but under conditions in which it is clear that the simultaneously presented cubes were perceived to reverse separately. Although some attentional effect might have been obtained if a greater number of cubes had been used, the findings remain consistent with the hypothesis derived from fatigue theory that reversals are mediated by multiple, relatively independent, local processes that can operate in parallel with one another while producing relatively little interference.

\section{EXPERIMENT 2}

In this experiment, we sought converging evidence for the hypothesis that reversible-figure illusions are due to the fatigue and recovery of neural channels. We again examined reversals of a rotating Necker cube in the context of an adaptation paradigm. However, adaptation and test cubes were presented to the same visual field, and we attempted to manipulate the involvement of channels by either varying or holding constant the size of the rotating Necker cube. On the assumption that the perception of different-sized rotating cubes involves the operation of different sets of cortical channels, we expected the reversal rate of the test cube to be elevated following adaptation to the same-sized cube but not following adaptation to the different-sized cube.

\section{Method}

Subjects. The 96 subjects were students at Villanova University who participated in partial fulfillment of the requirements of a general psychology course. Twenty-four subjects were assigned randomly to each of four viewing conditions.

Apparatus and Procedure. The apparatus and procedure were the same as those used in Experiment 1 with the following exceptions. First, cubes of two different sizes were employed. At a distance of $1 \mathrm{~m}$, the larger cube cast a shadow that was approximately $3.5^{\circ}$ on a side, whereas the smaller cube's shadow was approximately $1.5^{\circ}$ on a side. Second, subjects always saw and reported reversals on only one cube at a time. Third, although the size of the cube might change from the adaptation phase to the test phase of each 4-min viewing block, all cubes were presented to the same visual field for a given subject.

Viewing conditions. Each subject served in one of the following four viewing conditions. Within each condition, cubes were presented to the left visual field for half of the subjects and to the right visual field for the remaining subjects.

Lg-Lg. Subjects viewed the large cube during both the adaptation and test phases of each 4-min viewing block.

$L g-S m$. Subjects viewed the large cube during the adaptation phase and the small cube during the test phase.

$S m-L g$. Subjects viewed the small cube during the adaptation phase and the large cube during the test phase.

$\mathrm{Sm}-\mathrm{Sm}$. Subjects viewed the small cube during both the adaptation and test phases.

\section{Results and Discussion}

Effect of cube size. Several previous studies (e.g., Borsellino et al., 1982; Dugger \& Courson, 1968; Washburn et al., 1931) have compared reversal rates for stationary Necker cubes differing in size. Although the purposes of these studies varied somewhat, their results all indicated that reversal rate was inversely related to cube size; that is, reversal rate decreased as cube size increased. Our first analyses were intended to determine whether this wellestablished size effect extended to our rotating Necker cube procedure. The number of perceived reversals reported by subjects was analyzed for cubes presented to the left and to the right visual fields, respectively, by means of separate 2 (cube size: large vs. small) $\times$ 4 (interval: successive 30 -sec intervals) ANOVAs with repeated measures on the second factor. In each case, data were taken only from the adaptation phase of each 4-min viewing period in order to avoid confoundings with adaptation effects.

The results are presented in Figure 6 in which it is obvious that subjects perceived more reversals when viewing a small rather than a large rotating cube. This effect was reliable for both the left cube $[F(1,46)=30.34$, $p<.001]$ and the right cube $[F(1,46)=16.97$, $p<.001]$. In addition, the number of perceived reversals increased with successive 30 -sec viewing intervals for both the left and right cubes $[F s(3,138)=20.23$ and $11.76, p<.001$, respectively]. For the left cube, however, the cube-size $\times$ interval interaction was significant $[F(3,138)=3.74, p<.05]$. The interaction apparently was due to the fact that the increase in reported reversals between the first and second 30-sec intervals was greater for the small cube than for the large cube. We have no explanation for this effect, and it seems to be of little importance. The interaction does not alter our con-

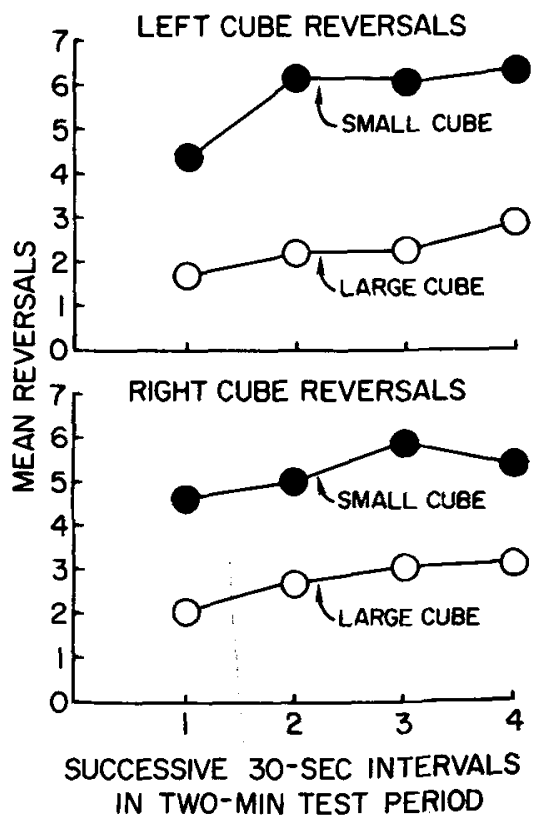

Figure 6. Mean reversals reported for a rotating Necker cube in Experiment 2 as a function of cube size (large vs. small) and 30-sec intervals constituting a 2 -min viewing period. 
clusions regarding the effect of cube size, and the same interaction failed to approach significance for the right cube $(F<1.00)$.

Adaptation effects. The primary purpose of this experiment was to determine how perceived reversals of a rotating Necker cube were influenced by adaptation to a cube of the same size appearing in the same retinal location or to a different-sized cube presented to the same visual field. The relevant analyses were conducted separately for the large and small cubes because of the large differences attributable to cube size that were revealed in the analyses described above.

In the first set of analyses, the number of perceived reversals obtained following adaptation to a cube of a different size (different adaptation) was compared with the number obtained without prior exposure to a cube (no adaptation). The results are presented Figure 7 . For the small cube, the different-adaptation data were taken from the test phase of the $\mathrm{Lg}-\mathrm{Sm}$ group, and the no-adaptation data were taken from the adaption phase of the Sm-Lg and Sm-Sm groups. For the large cube, the different-adaptation data were taken from the test phase of the Sm-Lg group, and the no-adaptation data were taken from the adaptation phase of the $\mathrm{Lg}-\mathrm{Lg}$ and $\mathrm{Lg}-\mathrm{Sm}$ groups. The small- and large-cube data were analyzed by means of separate 2 (adaptation condition: different vs. no adaptation) $\times 4$ (interval: successive 30 -sec intervals) ANOVAs with repeated measures on the second factor. In both analyses, the number of perceived reversals increased significantly as a function of successive 30 -sec intervals $[F \mathrm{~s}(3,210)=17.31$ and $20.60, p s<.001]$, for small and large cubes, respectively. More importantly, adaptation to a different cube did not influence performance for cubes of either size. That is, in comparison to the no-adaptation conditions, perceived reversals of a small cube were not influenced significantly by prior exposure to a large cube $[F(1,70)=1.27, p>.10]$, and perceived reversals of a large cube were not influenced significantly by prior

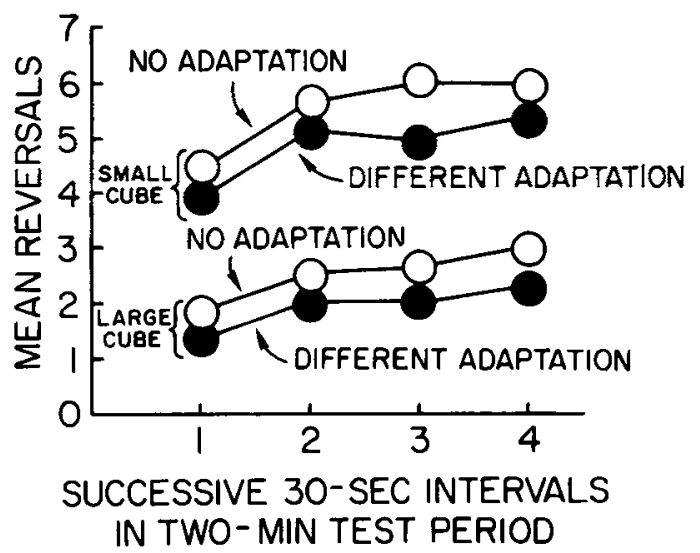

Figure 7. Mean reversals reported for a rotating Necker cube in Experiment 2 as a function of adaptation condition (different vs. no adaptation) and 30-sec intervals constituting a 2 -min viewing period.

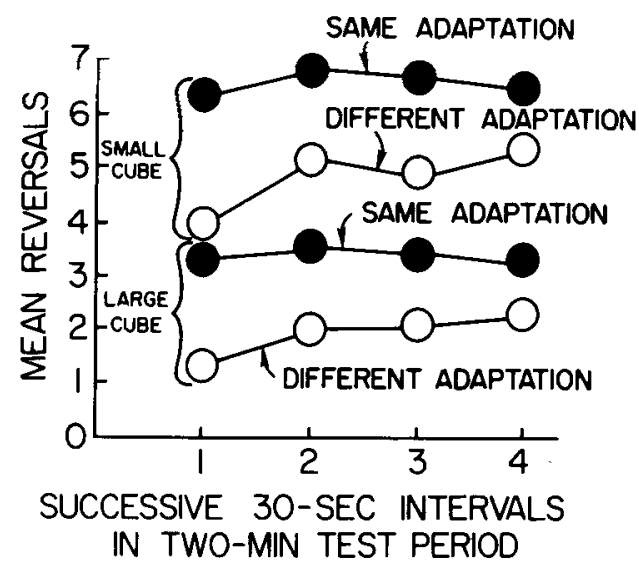

Figure 8. Mean reversals reported for a rotating Necker cube in Experiment 2 as a function of adaptation condition (same vs. different) and 30-sec intervals constituting a 2 -min viewing period.

exposure to a small cube $[F(1,70)=2.53, p>.10] . F i-$ nally, the adaptation condition $\times$ interval interaction was not reliable for either the small or the large cube $\left[F_{\mathrm{s}}(3,210)<1.05, p>.10\right]$.

In the second set of analyses, the effect of adaptation to the same-sized cube (same adaptation) was compared with the effect of adaptation to the different-sized cube (different adaptation). The data for the same-adaptation conditions were taken from the test phase of the Sm-Sm and the $\mathrm{Lg}-\mathrm{Lg}$ groups for the small and large cubes, respectively. Data for the different-adaptation conditions were the same as in the immediately preceding analyses. The results are presented in Figure 8. The data for the small cube and the large cube were submitted to separate 2 (adaptation condition: same vs. different) $\times 4$ (interval: successive 30-sec viewing intervals) ANOVAs with repeated measures on the second factor. These analyses indicated that the number of perceived reversals reported increased with successive 30-sec viewing intervals for both the small cube $[F(3,138)=6.47]$ and the large cube $[F(3,138)=4.35$, both $p s<.01]$. More importantly, inspection of Figure 8 reveals that the same-adaptation condition produced more perceived reversals than did the different-adaptation condition, with the difference between conditions being greatest in the beginning of the 2-min test phase and diminishing with successive $30-\mathrm{sec}$ intervals. These trends were supported by the fact that both the main effect of adaptation and the adaptation $\times$ interval interaction were significant $(p s<.05)$ for the small cube $[F(1,46)=7.54$, and $F(3,138)=3.31$, respectively $]$ and for the large cube $[F(1,46)=8.42$, and $F(3,138)=$ 2.77 , respectively]. Analyses of simple main effects indicated that both interactions reflected the fact that same adaptation produced reliably more reversals than different adaptation in the first three 30-sec intervals of the 2min test phase $\left[F_{\mathrm{s}}(1,62) \geq 6.46, p<.05\right.$, for the small cube, and $F_{\mathrm{s}}(1,61) \geq 6.57, p<.05$, for the large cube] (see Note 1). However, the difference was not reliable 
in the fourth $30-\mathrm{sec}$ interval for either the small cube $[F(1,62)=2.50, p>.05]$ or the large cube $[F(1,61)=$ $3.37, p>.05]$.

In conjuction with Experiment 1, the present experiment provided converging evidence in support of the hypothesis that the reversals of ambiguous figures are due to the fatigue and recovery of multiple, independent cortical channels. We attempted to control the neural channels mediating reversals by varying the size of rotating Necker cubes within the same visual field instead of by varying the visual field to which cubes of a constant size were presented as in Experiment 1. Interestingly, the results replicated the essential findings of Experiment 1. If the size of the cube (and, therefore, the underlying cortical channels) were changed from the adaptation to the test period, test-phase performance did not differ from that obtained without prior adaptation. Although the comparable effect was also nonsignificant in Experiment 1, there had been a trend in that experiment for the differentadaptation condition to yield more reversals than the noadaptation condition. This raises the possibility that the lack of a significant difference was due merely to insufficient power in our experiment. However, in Experiment 2 , there was actually a tendency for the nonsignificant difference to be in the opposite direction. Thus, when the results of both experiments are considered, the best conclusion appears to be that, within the limits of the sensitivity of our procedures, there is no reliable difference between the different-adaptation and the no-adaptation conditions. With regard to the effect of the same-adaptation condition, the results of Experiment 2 again paralleled those of Experiment 1. That is, if the size of the cube and the underlying cortical channels were the same in both the adaptation and test phases, reversal rate increased with viewing time during the adaptation period and remained at an asymptotic level during the test period. Thus, a different-sized cube reversed more slowly in the test period than did a same-sized cube. But, over the course of the test period, the reversal rate of the former increased, eventually approaching the asymptotic reversal rate of the latter.

It should be noted that one previous study (Cohen, 1959) examined the effect of size of a centrally fixated, stationary Necker cube in the context of an adaptation paradigm. The results were similar to ours in that the reversal rate during the test phase was higher following adaptation to the same-sized cube than following adaptation to a different-sized cube. However, Cohen's results differed from ours in that, relative to a no-adaptation condition, adaptation to a different-sized cube did produce some elevation in reversal rate during the test period. Why this discrepancy should exist is not clear, but there are at least several possibilities. First, channels in the periphery of the retina (our experiment) and in the fovea (Cohen's experiment) may differ in terms of their sensitivity or their interrelationships. Second, varying the size of a rotating cube may affect cortical channels that are not involved in the perception of a stationary cube. For example,
Beverley and Regan (1980; Petersik, Beverley, \& Regan, 1981; Regan \& Beverley, 1978) have proposed the existence of "changing-size" channels that might reasonably contribute to the perception of a rotating Necker cube but not to that of a stationary cube. Third, although the relative size of the front face of the cubes used by us and by Cohen were similar in terms of visual angle, it is not clear how comparable they were in the size or spatial location of other characteristics that actually might be more critical. For example, there is at least some evidence that certain corners of a stationary Necker cube are especially important in determining the perceived orientation of the figure (e.g., Kawabata, Yamagami, \& Noaki, 1978). These are the two corners that appear in the interior of the visual array (i.e., the corners that appear as the front, upper left corner and the rear, lower right corner of a cube perceived with its front face down and to the right). However, the relative location of these corners in two successively presented cubes of differing size does not depend on the length of the vertical or horizontal lines but rather on the length and the angle of the lines that run the "depth" of the cube. Although these corners did not occupy the same retinal location (assuming proper fixation) in Cohen's study, it is possible that they were sufficiently close together to fall at least partially within the spatial domain of some of the same sets of channels.

\section{CONCLUSIONS AND IMPLICATIONS}

The results of our experiments suggest that the perceptual reversals of rotating Necker cubes are mediated by the fatigue and recovery of multiple, largely independent neural channels, each sensitive to a particular form of stimulation in a particular region of the visual field. Thus, altering either the size of a cube or the visual field to which it is presented changes the neural channels mediating its perception and nullifies the effect of prior exposure. In addition, the channels seem to be capable of operating in parallel without discernible interference. Thus, the reversal rate of one rotating Necker cube is uninfluenced by the presence of a second cube, even under conditions in which the two cubes are reversing separately from one another.

The apparent involvement of neural channels in the perception of reversible figures suggests a theoretical link between reversible figure illusions and other visual phenomena. Consider, for example, the well-known tilt aftereffect. Inspection of a straight line whose orientation deviates from vertical by around $10^{\circ}$ in one direction results in a tendency to perceive a subsequently presented vertical line as tilted in the opposite direction (e.g., Magnussen \& Johnsen, 1986). Such an aftereffect often has been attributed to the satiation or fatigue of orientation channels during adaptation to the original stimulus. Moreover, recovery of the fatigued channels is hypothesized to account for the fact that the size of the aftereffect diminishes with time since the original stimulus was terminated. Similar aftereffects have been demonstrated with 
stimulus characteristics such as motion and size and are thought to be related to the fatiguing and recovery of channels sensitive to the relevant dimension of stimulation (e.g., channels sensitive to the direction of motion or to stimulus size). All in all, physiological and psychophysical studies have suggested the existence in the visual system of numerous channels that undergo fatigue and recovery similar to the processes that appear to underlie the perception of reversible figures. For example, in addition to channels for orientation, motion, and size (i.e., spatial frequency), there is evidence for channels attuned to stimulus characteristics such as changing size (e.g., Petersik et al., 1981; Regan \& Beverley, 1978), motionin-depth (Chase \& Smith, 1981; Regan \& Beverley, 1979), disparity (Schumer \& Ganz, 1979), and possibly others (e.g., see Bradick et al., 1978; Maffei, 1978; Regan, 1982).

Although our findings strongly indicate that the perception of reversible figures involves the fatigue and recovery of relatively localized neural channels, our results do not rule out the possibility that more global, decisional/learning mechanisms also may play a role under certain circumstances. In fact, it does not seem possible to ignore the evidence that the perception of reversible figures can be influenced by one's intentions and expectations (e.g., Girgus et al., 1977; Pelton \& Solley, 1968) as well as by learning (e.g., Adams, 1954; Ammons, 1954; Long et al., 1983).

As a number of investigators have suggested (e.g., Hochberg, 1981; Long et al., 1983; Palmer and Bucher, 1981; von Grünau et al., 1984), a full account of reversible-figure illusions may require some sort of multilevel theory involving both local and global processes. Thus, relatively local processes, such as those for which we have provided evidence in the present paper, might feed into and/or be coordinated by more global, perhaps decisional, mechanisms. Such a theoretical approach would help to reconcile our findings with results indicating that the perception of reversible figures can be influenced by global factors associated with configural and contextual characteristics of the visual array (e.g., Gillam, 1972; Palmer \& Bucher, 1981, 1982). This theoretical viewpoint also suggests that a major focus of future research on reversible figures should be on the nature of the interrelationships between local fatigue mechanisms and more global perceptual processes.

\section{REFERENCES}

ADAms, P. A. (1954). The effect of past experience on the perspective reversal of a tridimensional figure. American Journal of Psychology, 67, 708-710.

Ammons, R. B. (1954). Experiential factors in visual form perception: I. Review and formulation of problems. Journal of Genetic Psychology, 84, 3-25.

Ammons, R. B., UlRich, P., \& Ammons, C. H. (1959). Voluntary control of perception of depth in a two-dimensional drawing. Proceedings of the Montana Academy of Sciences, 19, 160-168.
Attneave, F. (1971). Multistability in perception. Scientific American, 225, 62-71.

Babich, S., \& Standing, L. (1981). Satiation effects with reversible figures. Perceptual \& Motor Skills, 52, 203-210.

BeVERLEY, K. I., \& Regan, D. (1980). Visual sensitivity to the shape and size of a moving object: Implications for models of object perception. Perception, 9, 151-160.

BoRING, E. G. (1942). Sensation and perception in the history of experimental psychology. New York: Appleton Century.

Borsellino, A., Caritni, F., Riani, M., Tuccio, M. T., De Marco, A., Penengo, P., \& Trabucco, A. (1982). Effects of visual angle on perspective reversal for ambiguous patterns. Perception, 11, 263-273.

Braddick, O., Campbell, F. W., Atkinson, J. (1978). Channels in vision: Basic aspects. In $R$. Held, H. W. Leibowitz, \& H. L. Teuber (Eds.), Handbook of Sensory Physiology (Vol. 8): Perception (pp. 339). Berlin: Springer-Verlag.

Brown, K. T. (1955). Rate of apparent change in a dynamic ambiguous figure as a function of observation time. American Joumal of Psychology, 68, 358-371.

CARLSON, V. R. (1953). Satiation in a reversible figure. Joumal of Experimental Psychology, 45, 442-448.

Chase, W., \& SMITH, R. (1981). Spatial frequency channels tuned for depth and motion. Visual Research, 21, 621-625.

COHEN, L. (1959). Rate of apparent change of a Necker cube as a function of prior stimulation. American Journal of Psychology, 72, 327-344.

DugGer, J. G., \& Courson, R. W. (1968). Effect of angle of retinal vision on the rate of fluctuation of the Necker cube. Perceptual \& Motor Skills, 26, 1239-1242.

Frissy, J. P. (1980). Seeing: Illusion, brain and mind. New York: Oxford University Press.

Gillam, B. (1972). Perceived common rotary motion of ambiguous stimuli as a criterion of perceptual grouping. Perception \& Psychophysics, 11, 99-101.

Girgus, J. J., Rock, I., \& Egatz, R. (1977). The effect of knowledge of reversibility on the reversibility of ambiguous figures. Perception \& Psychophysics, 22, 550-556.

GrEGORY, R. L. (1970). The intelligent eye. New York: McGraw-Hill.

HaRRIS, J. P. (1980). How does adaptation to disparity affect the perception of reversible figures? American Journal of Psychology, 93, 445-447.

HochbeRG, J. (1950). Figure-ground reversal as a function of visual satiation. Journal of Experimental Psychology, 40, 682-686.

HocHBERG, J. (1981). Levels of perceptual organization. In M. Kubovy \& J. R. Pomerantz (Eds.). Perceptual organization. Hillsdale, NJ: Erlbaum.

HowARD, I. P. (1961). An investigation of a satiation process in reversible perspective of revolving skeletal shapes. Quarterly Journal of Experimental Psychology, 13, 19-33.

Kawabata, N., Yamagami, K., \& Noaki, M. (1978). Visual fixation points and depth perception. Vision Research, 18, 853-854.

KoHler, W., \& Wallach, H. (1944). Figural aftereffects, an investigation of visual processes. Proceedings of the American Philosophical Society, 88, 269-357.

LoNG, G. M., \& TopPINo, T. C. (1981). Multiple representation of the same reversible figure: Implications for cognitive decisional interpretations. Perception, 10, 231-234.

Long, G. M., Toppino, T. C., \& Kostenbauder, J. F. (1983). As the cube turns: Evidence for two processes in the perception of a dynamic reversible figure. Perception \& Psychophysics, 34, 29-38.

MAFFEI, L. (1978). Spatial frequency channels: Neural mechanisms. In R. Held, H. W. Leibowitz, \& H. L. Teuber (Eds.), Handbook of sensory physiology (Vol. 8): Perception. Berdin: Springer.

MAGnussen, S., \& JoHNSEN, T. (1986). Temporal aspects of spatial adaptation. A study of the tilt aftereffect. Vision Research, 26, 661672.

MefFerd, R. B., JR., Wieland, B. A., CoOK, T. H., Sadier, T. G., Benton, R. G., \& ReDDING, G. M. (1966). Analysis of perspective 
reversal and associated apparent motions using a perspective-bound movement illusion. Perception \& Motor Skills, 22, 835-858.

PAlmer, S. E., \& Bucher, N. M. (1981). Configural effects on perceived pointing of ambiguous triangles. Journal of Experimental Psychology: Human Perception \& Performance, 7, 88-114.

Palmer, S. E., \& Bucher, N. M. (1982). Textual effects in perceived pointing of ambiguous triangles. Journal of Experimental Psychology: Human Perception \& Performance, 8, 693-708.

Pelton, L. H., \& Solley, C. M. (1968). Acceleration of reversals of a Necker cube. American Journal of Psychology, 81, 585-588.

Petersix, J. T., Beverley, K. I., \& Regan, D. (1981). Contrast sensitivity of the changing-size channel. Vision Research, 21, 829-832.

Price, J. R. (1969a). Effect of extended observation on reversible perspective duration. Psychonomic Science, 16, 75-76.

Price, J. R. (1969b). Studies of reversible perspective: A methodological review. Behavior Research Methods \& Instrumentation, 1, 102-106.

REgaN, D. (1982). Visual information channeling in normal and disordered vision. Psychological Review, 89, 407-444.

RegaN, D., \& BeVERLEY, K. I. (1978). Ilusory motion in depth: Aftereffects of adaptation to changing size. Vision Research, 18, 209-212.

REGAN, D., \& BEVERLEY, K. I. (1979). Binocular and monocular stimuli for motion in depth: Changing-disparity and changing-size feed the same motion-in-depth stage. Vision Research, 19, 1331-1342.
Rock, I. (1975). An introduction to perception. New York: Macmillan. SCHUMER, R., GANZ, L. (1979). Independent stereoscopic channels for different extents of spatial pooling. Vision Research, 19, 1303-1314. SPITZ, H. H., LiPMaN, R. S. (1962). Some factors affecting Necker cube reversal rate. Perceptual \& Motor Skills, 15, 611-625.

VIRSU, V. (1975). Determination of perspective reversals. Nature, 257, 786-787.

von Grünau, M. W., Wiggin, S., \& Reed, M. (1984). The local character of perspective organization. Perception \& Psychophysics, 35, 319-324

Washburn, M. F., Mallay, H., \& Naylor, A. (1931). The influence of the size of an outline cube on the fluctuations of its perspective. American Journal of Psychology, 43, 484-489.

\section{NOTE}

1. Degrees of freedom for the error term were derived by Satterthwaite's approximation.

(Manuscript received August 8, 1986; revision accepted for publication February 9, 1987.) 